\title{
Erratum to: A new computerized adaptive test advancing the measurement of health-related quality of life (HRQoL) in children: the Kids-CAT
}

\author{
J. Devine ${ }^{1}$ C. Otto ${ }^{1}$ - M. Rose ${ }^{2,3}$ - D. Barthel ${ }^{1}$ - F. Fischer ${ }^{2,4} \cdot$ H. Mühlan ${ }^{5}$. \\ S. Nolte ${ }^{2,6} \cdot$ S. Schmidt $^{5} \cdot$ V. Ottova-Jordan ${ }^{1}$ U. Ravens-Sieberer ${ }^{1}$
}

Published online: 19 March 2015

(c) Springer International Publishing Switzerland 2015

\section{Erratum to: Qual Life Res \\ DOI 10.1007/s11136-014-0812-7}

The name of the author 'H. Mülhan' was wrongly printed in the original publication of this article. The correct name of the author should be 'H. Mühlan'.

The online version of the original article can be found under doi:10.1007/s11136-014-0812-7.

\section{J. Devine}

janine.devine@web.de

U. Ravens-Sieberer

ravens-sieberer@uke.de;

http://www.child-public-health.de

1 Department of Child and Adolescent Psychiatry,

Psychotherapy, and Psychosomatics, University Medical

Center Hamburg-Eppendorf, Martinistr. 52, W29,

20246 Hamburg, Germany

2 Department of Psychosomatic Medicine and Psychotherapy, Charité-University Medicine Berlin, Sauerbruchweg 5, 10117 Berlin, Germany

3 Department of Quantitative Health Sciences, University of Massachusetts Medical School, Worcester, MA, USA

4 Institute for Social Medicine, Epidemiology and Health Economics, Charité University Medicine Berlin, Luisenstr. 57, 10117 Berlin, Germany

5 Institute of Psychology, Ernst-Moritz-Arndt Universität Greifswald, Robert-Blum-Str. 13, 17487 Greifswald, Germany

6 Population Health Strategic Research Centre, School of Health and Social Development, Deakin University, Burwood, VIC 3125, Australia 